\title{
Amplatzer Plugs versus Coil Embolization of the Hypogastric Artery Prior to Endovascular Aortic Aneurysm Repair: Differences in Quality of Life ${ }^{*}$
}

\author{
Luluel Khan ${ }^{1}$, Dheeraj K. Rajan ${ }^{2 *}$, Kong T. Tan ${ }^{2}$, Thomas F. Lindsay ${ }^{3}$ \\ ${ }^{1}$ Department of Radiation Oncology, University of Toronto, Toronto, Canada \\ ${ }^{2}$ Department of Medical Imaging, University of Toronto, Toronto, Canada \\ ${ }^{3}$ Department of Vascular Surgery, University of Toronto, Toronto, Canada \\ E-mail: *dheeraj.rajan@uhn.on.ca \\ Received September 13, 2011; revised October 6, 2011; accepted November 6, 2011
}

\begin{abstract}
Purpose: To determine if there were differences in quality of life (QOL) within the first year following EVAR for patients undergoing internal iliac embolization depending on the type of device used. Methods: Patients who underwent endovascular AAA repair were identified using a Vascular surgery database at a tertiary care center from 2002-2008. The Radiology Information System and Image Viewer were then used to identify patients who underwent preprocedural embolization prior to endovascular aneurysm repair. Nine patients had embolization with nester coils, 9 had embolization with the amplatzer vascular plug, a type of nitinol based self expanding device. Another group of 8 patients who did not undergo preprocedural embolization was used as a comparator group. These patients were contacted via telephone and answered questions regarding QOL post procedure. The Australian Vascular QOL was the tool used to measure QOL. Differences in QOL were tabulated between the groups of patients. Results: Of the 9 patients who underwent embolization with amplatzer plug, the median QOL score was 60 (p value 0.575), the median QOL for the nester coil group was 52.5 and the comparator group was 58. Separate analysis was done dividing patients into two groups, with and without comorbidities without statistical significance. Conclusion: Patients who underwent preprocedural embolization using amplatzer plugs compared to coils had higher overall QOL scores although the difference was not significant.
\end{abstract}

Keywords: Amplatzer Plug, Coil, Embolization, EVAR, AAA

\section{Introduction}

Patients with abdominal aortic aneurysms (AAA) that are high risk for open repair undergo endovascular aneurysm repair (EVAR) to reduce peri operative mortality. Approximately $10 \%-20 \%$ of AAA involve the iliac arteries [1]. Iliac aneurysm repair can be achieved by several methods including iliac branched grafts, occlusion of the internal iliac and coverage into the external iliac with the graft limb or surgical bypass to the internal iliac artery. Therapeutic embolization of the internal iliac arteries are required in up to $20 \%$ of patients with AAA extending to the common iliac arteries prior to EVAR [2,3].

Pre-procedural occlusions can be achieved using the conventional coils or nitinol based plugs. In 2005, Ha et

\footnotetext{
"Acknowledgements: Ravi Menezes PhD for statistical support.
}

al. showed that the use of Amplatzer plugs allow for a more cost effective, technically convenient method to occlude the internal iliac artery in patients undergoing EVAR for aortoiliac aneurysms [1]. Ischemic complications secondary to internal iliac occlusion include buttock claudication, sexual dysfunction and in some cases gluteal necrosis although rare. Buttock claudication can resolve in $25 \%-40 \%, 6-12$ months post procedure as collaterals develop [4]. Marty et al. stated that not all ischemic changes are secondary to perfusion changes but occur secondary to atheroembolization events during insertion of occlusion devices [5].

We hypothesized that the greater accuracy of plug placement compared to coil insertion may result in reduced morbidity associated with internal iliac artery occlusion. To assess this hypothesis, we conducted a qual- 
ity of life score one year post embolization. To our knowledge no study has compared the QOL scores amongst patients who underwent internal iliac occlusion using two different devices. The Australian Vascular QOL is a validated QOL assessment tool for patients with AAA in the clinical setting [6]. We used the assessment tool to delineate the difference in QOL scores between the two embolization patient groups and a comparator group.

\section{Methods}

A Vascular Surgery database at a tertiary care center was used to identify patients who underwent EVAR between 2002 and 2008 after research ethics approval was obtained. From this database 39 patients were identified. Out of the 39 patients; 9 had embolization with an Amplatzer nitinol plug, 10 had embolization with nester coils and 8 did not have embolization prior to repair. Of the 13 that were left, 4 patients died prior to being contacted for participation in the study. One patient died of multi-organ failure following EVAR for ruptured aneurysm, the other three patients died of unrelated causes. Six patients could not be reached for a variety of reasons, and the last three did not wish to participate in the survey. One patient from the Amplatzer group was excluded as they were immobile prior to the procedure as a result of spinal cord injury. The eighteen patients who underwent pre-procedural embolization with either device were asymptomatic with respect to buttock symptoms prior to EVAR repair and were being monitored routinely with ultrasound until they met size criteria for repair. The eight patients who did not undergo embolization prior to EVAR were used as a comparator group. These twenty six patients were contacted at post surgery and the AUSVIQUOL was used as measure of Quality of Life.

The maximum score for the Australian QOL assessment tool is 100. It is divided into the following sections: General Health Perceptions, Physical Domain and Psychosocial. These three domains are weighted differently with physical domain being given the greatest weight totaling 50 points, general health perceptions 30 points and psychosocial domain 20 points (Appendix). The assessment tool was administered by a research assistant not part of the vascular team, and not familiar to the patients. Data was collected and statistical analysis completed using Man Whitney and Chi square tests. A p value of less than 0.05 was considered significant.

\section{Technical Details}

With both devices, the endpoint for occlusion was proper positioning and observed reduction in flow into the internal iliac artery but not cessation of flow. Selection of device was operator preference and in select cases, technical issues (tortuosity of vessels, calcification of vessels, ipsilateral versus contralateral approach) that prevented proper placement of the delivery sheath within the internal iliac artery for proper plug deployment. In no instances, were coils used as a failure of plug deployment. Coils were placed using either a 4 or 5 Fr angled catheter within the origin of the internal iliac artery, stabilized by an outer guiding catheter or sheath and subsequent advancement of coils under fluoroscopic guidance using a Berenstein wire to push the coils into the artery. Initially, a large coil(s) was placed for wall apposition with subsequent filling of the coil mass with smaller coils to the point of visualized reduction in flow through the coil mass. Complete cessation of flow was not sought as a majority of coiled or blocked IIA's would thrombose prior to EVAR or thrombose immediately after stent graft placement over the internal iliac artery origin. For Amplatzer plug deployment, the appropriately sized guiding sheath was advanced into the origin of the internal iliac artery and the plug was deployed within the proximal portion of the artery.

\section{Results}

There were 22 males and 4 females in our study. The average ages of patients were 83, 81 and 76 for the Amplatzer, coil and comparator groups respectively. The oldest patient was 91 and the youngest was 64 . Of the 26 patients 18 had comorbidities. All embolization procedures were technically successful with no associated complications. All patients in the study also went on to have technically and clinically successful exclusion of their AAA's with EVAR. Follow-up CT's demonstrated no endoleaks originating from the embolized internal iliac arteries. Table 1 provides details regarding patient demographics.

The overall median QOL score for Amplatzer group was 60. The median scores for each individual domain for this group were as follows: general health perception 19, Mobility 32, Psychosocial 13.5. The overall median score for the coil group was 52.5. The median scores for each domain were 15, 25.5, 11 for general health perceptions, mobility and psychosocial domains respectively. No significant difference was observed between the embolization groups $(p=0.575)$. In the control group the overall median QOL was 58 with categorical scores of 14, 37, 9 in the same order as for above (Figure 1). No significant difference was observed compared to patients who underwent embolization ( $p=0.96$ ). Looking specifically at mobility, the Amplatzer group had a higher mobility score than the coil group (p value of 0.922 ), and the control group maintaining the highest mobility score. 
Table 1. Patient demographics.

\begin{tabular}{|c|c|c|c|}
\hline Patient Info & Coils & Plugs & No embolization \\
\hline Mean Age & 81 & 83 & 76 \\
\hline Gender & 8 Male, 1 Female & 8 Male, 1 Female & 6 Male, 2 Female \\
\hline Comorbidity & $\begin{array}{l}5 \text { patients } \\
\text { 1) HTN } \\
\text { 2) Gout } \\
\text { 3) Afib } \\
\text { 4) CABG } \\
\text { 5) Renal failure } \\
\text { *** } 2 \text { patients had gout, } 1 \text { patient } \\
\text { had gout AFIB and HTN }\end{array}$ & $\begin{array}{l}6 \text { patients } \\
\text { 1) HTN } \\
\text { 2) Renal failure } \\
\text { 3) Cancer }{ }^{* *} \\
\text { 4) Nephrolithiasis } \\
\text { 5) Gout } \\
\text { 6) Emphysema } \\
{ }^{* *} 1 \text { patient had cancer and HTN }\end{array}$ & $\begin{array}{l}7 \text { patients } \\
\text { 1) HTN } \\
\text { 2) MI } \\
\text { 3) CAD } \\
\text { 4) Cancer* } \\
\text { 5) Renal insufficiency } \\
\text { 6) Paralyzed T6 and below } \\
\text { 7) Endarterectomy } \\
\text { 8) DM } \\
\text { *1 patient had cancer and renal } \\
\text { insufficiency }\end{array}$ \\
\hline Median QOL scores & 52.5 p 0.55 & 60 p 0.96 & 58 p 0.96 \\
\hline
\end{tabular}

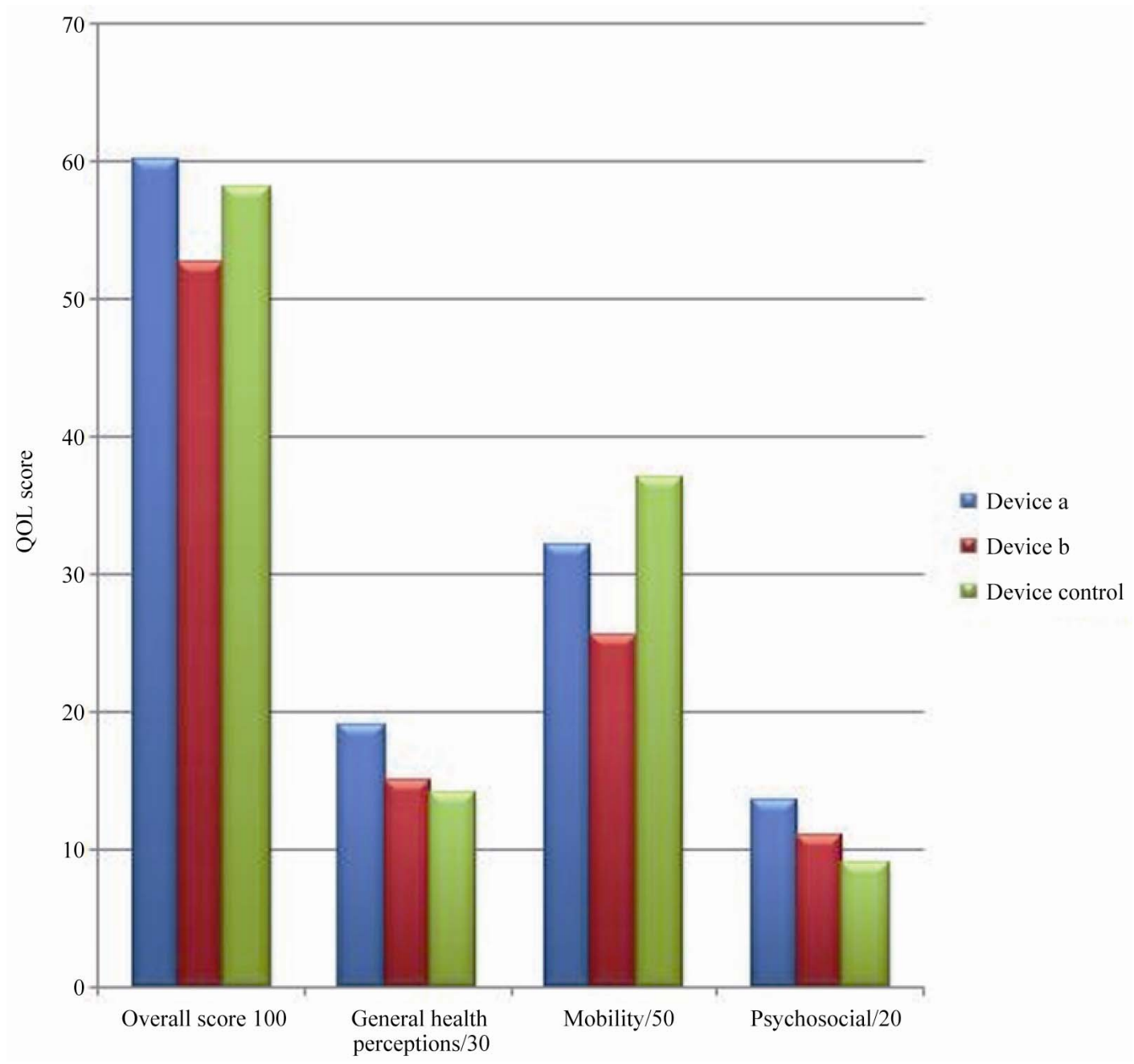

Figure 1. Median QOL scores for each domain of the AUSVIQUOL (Device a-Amplatzer Plug, Device b-coils, Device control-no embolization). 
Eleven patients in the pre-occlusion group had comorbidities whilst eight patients had no comorbidities, some had multiple. Those who did not have comorbidities had median QOL score of 62.5 and those with comorbidities had a median QOL score of 55 (p value 0.66). The patient with the lowest overall QOL score was in the Amplatzer group was a palliative patient with advanced cancer and thus had a significantly lower QOL score.

All patients who had embolizations with coils had multiple coils used. The least number of coils used was 2 , with a maximum of 15 coils per procedure. The average number of coils used per procedure was 7.6. The cost of each coil was noted to be approximately $\$ 149$. The average procedure time for the Amplatzer and coil group was 2.44 hours and 3 hours respectively $(p=0.29)$. Procedure time began at the time the patient entered the room and departed the room and is not specific to the embolization procedure itself. In addition, some of the procedures were performed intraoperatively and include the EVAR. The patients who had embolizations with the Amplatzer plug on average used one plug with the use of two plugs if more than one internal iliac artery was being occluded. The cost of each plug is approximately $\$ 675$. Since an average of 7.6 coils were used per procedure with a total cost of approximately \$ 1132 .

\section{Discussion}

The Medical Research Short Form survey SF-36 has been the gold standard for QOL research [7]. However this is a very generic assessment tool not specific to any patient population. Other QOL measures such as the WHOQOL and the CLAUS-S scale were also considered $[8,9]$. The AUSVIQUOL developed by Borchard et al. in 2006 is the first disease specific QOL tool for AAA patients in the clinical setting. This QOL assessment tool was intended for patients who had vascular surgery and not the general population. In addition, it was also catered to the elderly population which is why there was a question regarding frequency of feeling lonely. It also has additional questions regarding complications from vascular surgery that the general patient population would not experience. In the follow up study to validate the AUSVIQUOL in 2007 Smith et al. showed that AUSVIQUOL was an improved tool for measuring quality of life in vascular patients [10]. Since this was a validated tool for measuring QOL scores in the AAA population, we chose this survey as a measure of QOL in our patients. To our knowledge, no study has addressed the question of whether there is a difference in quality of life depending on the type of device used for embolization.

Our results show that embolization with the Amplatzer plug results in a better overall QOL score than using the coils, although the result was not found to be statistically significant. We believe that statistical significance could be reached with a larger sample size. Currently our study is powered at $8 \%$ with an alpha of 0.05 . It is interesting to note that patients in the Amplatzer group had higher scores in every domain compared to the coil group. In addition, the Amplatzer group also had higher scores in the general health perceptions and psychosocial domain than the comparator group. For example, in the mobility domain, which is of particular interest in this population, the score in the control group was highest, followed by the plug group, although not statistically significant ( $\mathrm{p}=$ 0.922). This could be attributable to the fact that patients who did not have embolization had the most comorbidities and perhaps had poor general health perceptions, which is a subjective measure compared to mobility section which is a more objective measure, for which they had higher scores than the Amplatzer and coil group. Aquarius et al. showed that high levels of perceived stress adversely affect QOL in addition to health status and walking ability in patients with peripheral vascular disease beyond the scope of clinical indicators [11]. The intervention groups are comparable as they were similar in baseline demographics and had the same procedure, with similar technical outcomes but utilizing different devices. Approximately 66\% of patients in the plug group had comorbidities compared to $55 \%$ the coil group and $88 \%$ in the control group. Thus we can see that comorbidities did not have an effect on QOL scores.

If the use of Amplatzer plug truly does provide an improved quality of life compared to the use of other devices, and similar scores compared to the control group, than this would be an added reason for using plugs over coils. The plugs are not only cheaper but are also easier to position accurately within the main trunk of the internal iliac artery when technically possible, thereby preserving collateral flow between the anterior and posterior divisions. There is also less of a tendency for them to move compared to the coils [12]. Coil embolization due to technical limitations is more likely to result in a more distal embolization of the internal iliac artery which may prevent collateral flow between the anterior and posterior divisions thereby leading to increased risk of gluteal claudication.

Our study has limitations including its' retrospective nature and the sample size was relatively small. QOL results may have been influenced by recall bias as patients were asked to answer questions about their well being post intervention. This issue could be addressed in the future by conducting the survey immediately post intervention to minimize recall bias. Also in addition, it would be interesting to do the survey pre and post procedure to compare the results between the two groups. 
Prospective study with a larger patient cohort would be required to determine if our findings are statistically significant.

In conclusion, although there was no significant difference in QOL scores between Amplatzer plug and coil embolization groups, the Amplatzer group had overall improved QOL scores across all categories with a trend towards significance.

\section{References}

[1] C. Ha and D. Calgano, "Amplatzer Vascular Plug to Occlude the Internal Iliac Arteries in Patients Undergoing Aortoiliac Aneurysm Repair,” Journal of Vascular Surgery, Vol. 42, No. 6, 2005, pp. 1058-1062.

[2] M. Armon, P. Wenham, S. Whitaker, R. Gregson and B. Hopkinson, "Common Iliac Artery Aneurysms in Patients with Abdominal Aortic Aneurysms,” European Journal of Vascular and Endovascular Surgery, Vol. 15, No. 3, 1998, pp. 255-257.

[3] F. Criado, "Iliac Bifurcation Relocation: More Complex and Controversial," Journal of Endovascular Surgery, Vol. 6, No. 4, 1999, pp. 348-349.

[4] L. A. Karch, K. J. Hodgson, M. A. Mattos, W. T. Bohannon, D. E. Ramsey and R. B. Mclafferty, “Adverse Consequences of Internal Iliac Artery Occlusion during Endovascular Repair of Abdominal Aortic Aneurysms," Journal of Vascular Surgery, Vol. 32, No. 4, 2000, pp. 676-683.

[5] B. Marty, C. Perrouchould, S. Wicky, L. Guilou and L. K. Von Segesser, "Atheroembolization: A Harmful Complication of therapeutic Internal Iliac Artery Occlusion," Journal of Vascular Surgery, Vol. 35, No. 5, 2002, pp. 1062-1065.
[6] K. Borchard, P. Hewitt, S. Wotherspoon and A. R. Scott, "Australian Vascular Quality of Life Index (AUSVIQUOL): A Pilot Study of a Disease-Specific Quality of Life Measure,” ANZ Journal of Surgery, Vol. 76, No. 4, 2006, pp. 208-213.

[7] C. Shearman, In: J. J. Earnshaw, J. A. Murie, Eds., The Evidence for Vascular Surgery, TFM Publishing Limited, Shrewsbury, UK, 2006, pp. 37-44.

[8] WHOQOL Group, “The World Health Organization Quality of Life Assessment (WHOQOL): Position Paper from the World Health Organization,” Social Science \& Medicine, Vol. 41, No. 10, 1995, pp. 1403-1409.

[9] F. A. Spengel, T. M. Brown, S. Dietze, I. Kirchberger and S. Comte, "The Claudication Scale (CLAU-S): A New Disease-Specific Quality of Life Instrument in Intermittent Claudication," Disease Management and Health Outcomes, Vol. 2, Supplement 1, 1997, pp. 65-70. doi:10.2165/00115677-199700021-00013

[10] M. J. Smith, K. L. Borchard, E. Hinto and A. R. Scott, "Australian Vascular Quality of Life Index (AUSVIQUOL): An Improved Clinical Quality of Life Tool for Peripheral Vascular Disease,” European Journal of Vascular and Endovascular Surgery, Vol. 34, No. 2, 2007, pp. 199-205. doi:10.1016/j.ejvs.2007.02.005

[11] A. Aquarius, J. Vries, D. Van Berge Henegouwen and J. F. Hamming, "Clinical Indicators and Psychosocial Aspects in Peripheral Arterial Disease,” Archives of Surgery, Vol. 141, No. 2, 2006, pp. 161-166. doi:10.1001/archsurg.141.2.161

[12] F. Vandy, E. Criado, R. U. Gilbert, D. M. Williams, J. Rectenwald and J. Eliason, "Transluminal Hypogastric Artery Occlusion with an Amplatzer Vascular Plug during Endovascular Aneurysm Repair,” Journal of Vascular Surgery, Vol. 48, No. 5, 2008, pp. 1121-1124. doi:10.1016/j.jvs.2008.06.002 


\section{Appendix}

The Australian Vascular Quality of Life Index (adopted from Smith et al.)

\section{General Health Perceptions}

Q1. How has your health been in the last month compared to other people you know of the same or similar age?

Excellent

Very Good

Good

Fair

Poor

Q2. During the last month how many days have you spent, sick in bed or sitting in a chair?

None

Between 1 and 5 days

Between 6 and 14 days

Between 15 and 30 days

Everyday

If you had vascular surgery go to 3a, if not go to $3 b$

Q3a. In comparison to your health before vascular surgery, how would you rate your health now?

A lot better than before the surgery

A little better than before the surgery

The same as before the surgery

A little worse than before the surgery

A lot worse than before the surgery

Q3b. In comparison to your health 1year ago, how would you rate your health now?

A lot better than 1 year ago

A little better than 1 year ago

The same as 1 year ago

A little worse than 1 year ago

A lot worse than 1 year ago

Function, Mobility and Pain

Q4. At a steady pace how far are you able to walk on the flat before becoming short of breath, experience chest pain, leg pain or another limitation?

More than $1 \mathrm{~km}$

Between $500 \mathrm{~m}$ and $1 \mathrm{~km}$

Between $100 \mathrm{~m}$ and $500 \mathrm{~m}$

Between $1 \mathrm{~m}$ and $100 \mathrm{~m}$

I can't walk, have pain at rest

Q5. Do you have pain and discomfort in your legs and feet that limits your mobility or disturbs your sleep, ulcers on your feet or have you lost a limb?

None of these

Leg or foot pain when I walk

Leg or foot pain at night
I have an ulcer on my foot or leg

I have had a limb(s) amputated

Q6. Are you able to do most of the chores around the house and do your grocery shopping?

Yes, easily

Yes, but I find it difficult

Yes, with some assistance

Yes with continuous assistance

No, not at all

Q7. Do you suffer from fits, faints, funny turns, memory problems (including epilepsy, TIA, episodes of dizziness, loss of consciousness or stroke?)

Never/I used to/I had a non-disabling stroke

Occasionally

Sometimes

Often

Continuously/I have had a disabling stroke

Q8. Are you able to read a magazine or newspaper?

Yes, easily

Yes, but I find it difficult

Yes, with prescription glasses

No, but I still have some sight

No, I am blind

Psychosocial Aspects

Q9. How often do you see your friends and relatives and participate in hobbies?

Everyday

Several times per week

Once per week

Several times per month

Rarely/Never

Q10. Have you felt lonely, unhappy, anxious or depressed over the past month?

No

Yes, occasionally

Yes, sometimes

Yes, often

All the time

\section{AUSVIQUOL Scoring Sheet}

$$
\begin{aligned}
& \text { Scoring Q1 - Q10: } \\
& \begin{array}{l}
\text { Response } \\
\mathrm{a}=10 \text { points } \\
\mathrm{b}=5 \text { points } \\
\mathrm{c}=2 \text { points } \\
\mathrm{d}=1 \text { point } \\
\mathrm{e}=0 \text { point }
\end{array}
\end{aligned}
$$

Domain Scores

General Health Perceptions/30

Mobility/50

Psychosocial Aspects/20 
Total/100

This person's quality of life is:

Excellent.........100

Very Good.........80 - 99
Good..............55 - 79

Fair................21 - 54

Poor................ 0 - 20 